\title{
VMAT2-Deficient Mice Display Nigral and Extranigral Pathology and Motor and Nonmotor Symptoms of Parkinson's Disease
}

\author{
Tonya N. Taylor, ${ }^{1,2,3}$ W. Michael Caudle, ${ }^{1,2}$ and Gary W. Miller ${ }^{1,2,4,5}$ \\ ${ }^{1}$ Department of Environmental Health, Rollins School of Public Health, Emory University, \\ 1518 Clifton Road, Atlanta, GA 30322, USA \\ ${ }^{2}$ Center for Neurodegenerative Disease, Rollins School of Public Health, Emory University, \\ 1518 Clifton Road, Atlanta, GA 30322, USA \\ ${ }^{3}$ Department of Physiology, Anatomy and Genetics, University of Oxford, Oxford OX1 3QX, UK \\ ${ }^{4}$ Department of Neurology, Rollins School of Public Health, Emory University, 1518 Clifton Road, Atlanta, GA 30322, USA \\ ${ }^{5}$ Department of Pharmacology, Rollins School of Public Health, Emory University, 1518 Clifton Road, Atlanta, GA 30322, USA
}

Correspondence should be addressed to Gary W. Miller, gwmille@emory.edu

Received 27 October 2010; Accepted 3 January 2011

Academic Editor: Huaibin Cai

Copyright ( $\odot 2011$ Tonya N. Taylor et al. This is an open access article distributed under the Creative Commons Attribution License, which permits unrestricted use, distribution, and reproduction in any medium, provided the original work is properly cited.

\begin{abstract}
Dopamine is transported into synaptic vesicles by the vesicular monoamine transporter (VMAT2; SLC18A2). Disruption of dopamine storage has been hypothesized to damage the dopamine neurons that are lost in Parkinson's disease. By disrupting vesicular storage of dopamine and other monoamines, we have created a progressive mouse model of PD that exhibits catecholamine neuron loss in the substantia nigra pars compacta and locus coeruleus and motor and nonmotor symptoms. With a 95\% reduction in VMAT2 expression, VMAT2-deficient animals have decreased motor function, progressive deficits in olfactory discrimination, shorter latency to behavioral signs of sleep, delayed gastric emptying, anxiety-like behaviors at younger ages, and a progressive depressive-like phenotype. Pathologically, the VMAT2-deficient mice display progressive neurodegeneration in the substantia nigra (SNpc), locus coeruleus (LC), and dorsal raphe (DR) coupled with $\alpha$-synuclein accumulation. Taken together, these studies demonstrate that reduced vesicular storage of monoamines and the resulting disruption of the cytosolic environment may play a role in the pathogenesis of parkinsonian symptoms and neurodegeneration. The multisystem nature of the VMAT2deficient mice may be useful in developing therapeutic strategies that go beyond the dopamine system.
\end{abstract}

\section{Introduction}

Parkinson's disease (PD) is a devastating neurodegenerative disease and is characterized by a preferential loss of dopamine neurons. PD is distinguished by the cardinal symptoms of resting tremor, rigidity, bradykinesia, and postural instability [1-3]. The incidence of PD is positively correlated with age; there is a greater than 40 -fold increase in prevalence between the ages of 55 and 85 [3]. Approximately 5-10\% of PD patients have a familial form of Parkinsonism with either an autosomal dominant or autosomal recessive pattern of inheritance. These familial forms are characterized by an age of onset before 40 years and a slowly progressive course [4]. Pathogenic changes in PD are extensive and, in addition to the loss of dopaminergic neurons in the substantia nigra pars compacta ( $\mathrm{SNpc}$ ) and loss of striatal innervation, include degeneration of the norepinephrine (NE) neurons of the locus coeruleus (LC), serotonin (5-HT) neurons of the raphe nuclei, the dorsal motor nucleus of the vagus, and the peripheral autonomic nervous system, among others $[3,5,6]$. Furthermore, Lewy body pathology can also be found in the LC, nucleus basalis of Meynert, hypothalamus, cerebral cortex, and in components of the peripheral nervous system $[2,3,7]$. As the acknowledgement of pathology associated with PD expands, symptoms beyond the cardinal motor phenotype are also more commonly recognized, including hyposmia, sleep disturbances, gastrointestinal dysfunction, anxiety, depression, and autonomic disturbances $[8,9]$. The onset of these nonmotor symptoms typically comprise a prodromal phase of the disease, which 
can last anywhere from a few years to decades. These symptoms often play a large role in the quality of life and disease etiology, and highlight the need to be more vigilant as we look beyond a dopamine centric view and broaden our understanding of PD pathogenesis. In doing so, targets for therapeutic intervention may be revealed and provide a more comprehensive view of the disorder.

Abnormalities with monoaminergic handling and neurotransmission are associated with a number of neurological disorders, in addition to PD, such as schizophrenia, depression, and drug addiction. Although the etiopathogenesis of PD remains unclear, it has been hypothesized that the mishandling of DA as well as other monoamines could underlie disease development. In this regard, many researchers have proposed that the accumulation of cytosolic DA has the ability to induce cytotoxicity with age; however, the long-term toxicity of DA in vivo has only recently been firmly established [10]. Many chemical models of PD, such as 6-OHDA, manipulate the oxidative environment of dopaminergic neurons to induce cell death. The endogenous generation of reactive oxygen species (ROS), resulting from both metabolism of monoamines in the cytosol and autooxidation of monoamines, has been implicated as a mediator in the pathophysiology of PD $[10,11]$. However, physiologically, neurons have many safeguards to maintain neuronal health and protect against degeneration.

The vesicular monoamine transporter 2 (VMAT2) is one such custodian that functions to regulate the cytosolic environment of the neuron, protecting it from endogenous and exogenous toxins. Localized on vesicular membranes in neurons, VMAT2 acts to accumulate cytosolic monoamines into synaptic vesicles after they have been synthesized from their precursors for regulated exocytotic release [12]. The sequestration of monoamines is important for maintenance of normal neurotransmission and also acts to keep intracellular levels of the monoamines below potentially toxic levels $[13,14]$. VMAT2 is a 12 -transmembrane domain $\mathrm{H}^{+}$-ATPase antiporter, which uses an electrochemical gradient to drive transport; two protons are exchanged for one monoamine molecule $[13,15,16]$. VMAT2 has a similar selectivity for all monoamines and is present throughout the central nervous system and in the periphery in mast cells and platelets. Phylogenetically, VMAT2 is a member of the solute carrier protein family and the toxin-extruding antiporter (TEXAN) gene family, which includes bacterial resistance genes [17, 18]. Moreover, VMAT2 contains sequence homology and functional similarities to the major facilitator superfamily of drug resistance transporters; many researchers have hypothesized that VMAT2 has evolved to serve an analogous role in eukaryotic systems by providing a mechanism to sequester and clear toxins from the cell [19, 20]. Thus, vesicular sequestration serves a dual purpose: preventing the interaction of toxins with molecular machinery and limiting exposure of neighboring cells to the toxin. In fact, VMAT2 was partly identified via its ability to confer resistance to the dopaminergic toxin 1-methyl-4-phenylpyridinium $(\mathrm{MPP}+)$, which is commonly used to induce a Parkinsonian phenotype in mice [14]. The level of VMAT2 expression is essential to proper monoaminergic handling, as it regulates both the size of the vesicular monoamine pool and influences the availability of monoamines in the cytosol, influencing cellular susceptibility to oxidation [14]. The monoamines, particularly DA and norepinephrine (NE) have the ability to spontaneously oxidize in the cytosol, potentially damaging cellular machinery [21].

\section{VMAT2 and PD}

Evidence for the monoamine theory of PD surfaced as early as the 1950s but has not begun to be fully appreciated until recently. Reserpine, an inhibitor of vesicular monoamine transport, was first introduced as a potent antihypertensive drug [22]. Reserpine acts by depleting cells of their monoamine stores; however, it is not selective for the periphery and affects the central nervous system as well [22, 23]. Patients who took reserpine chronically began to display lethargy similar to that seen in depression, contributing to the monoamine hypothesis of affective disorders [22]. Furthermore, treatment with reserpine also reproduced many of the deficits observed in $\mathrm{PD}$, including a decrease in locomotor activity, akinesia, L-DOPA responsive stride length, a depressive-like phenotype, and cognitive decline [24-27]. Acute depletion of monoamine stores was found to reproduce a similar symptom profile as mice dosed with the commonly used MPTP model of PD.

Theoretically, the loss of VMAT2 function within the neuron would be associated with a reduction in vesicular sequestration of monoamines, a concomitant accumulation of cytosolic monoamines, depletion of striatal monoamines, and the development of a Parkinsonian phenotype. It is thought that together with the dopamine transporter (DAT), VMAT2 may be able to modulate susceptibility to neurodegeneration $[20,28]$. There has been much speculation about the role of VMAT2 in mediating efficient clearance of DA in those populations vulnerable to neurodegeneration $[28,29]$. To this end, a positive correlation exists between VMAT2 expression levels and regions of the brain spared from Parkinsonian degeneration. For example, the caudate and putamen have higher DAT expression relative to VMAT2, consequently incurring more damage than other monoaminergic areas of the brain like the hypothalamus, which has high levels of VMAT2 relative to DAT [20]. In vivo imaging and postmortem-binding studies displayed marked reductions in VMAT2 immunoreactivity in the caudate, putamen, and nucleus accumbens of PD brains [30, 31]. Interestingly, a gain of function haplotype of VMAT2 was found to be protective against the development of PD in humans [32]. Despite these data, it is still unclear if reductions in VMAT2 are a contributor to or a consequence of PD.

VMAT2 has been directly implicated with a pathological hallmark of PD: $\alpha$-synuclein. This key component of Lewy bodies has been found to bind and permeabilize vesicles, potentially causing leakage of monoamines into the cytosol [33]. This has been hypothesized to be mediated via a direct interaction between VMAT2 and $\alpha$-synuclein, disrupting synaptic vesicle dynamics [34]. Moreover, overexpression of 


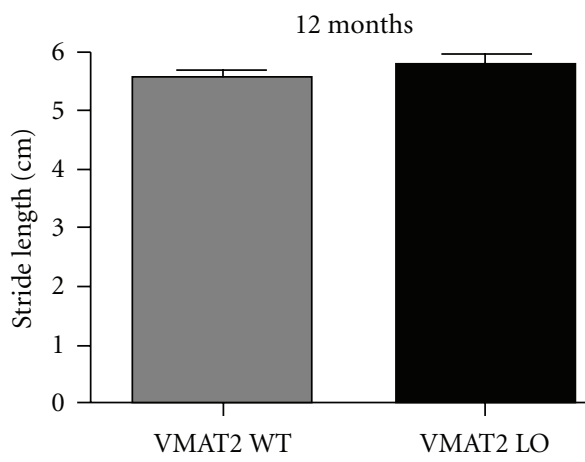
VM
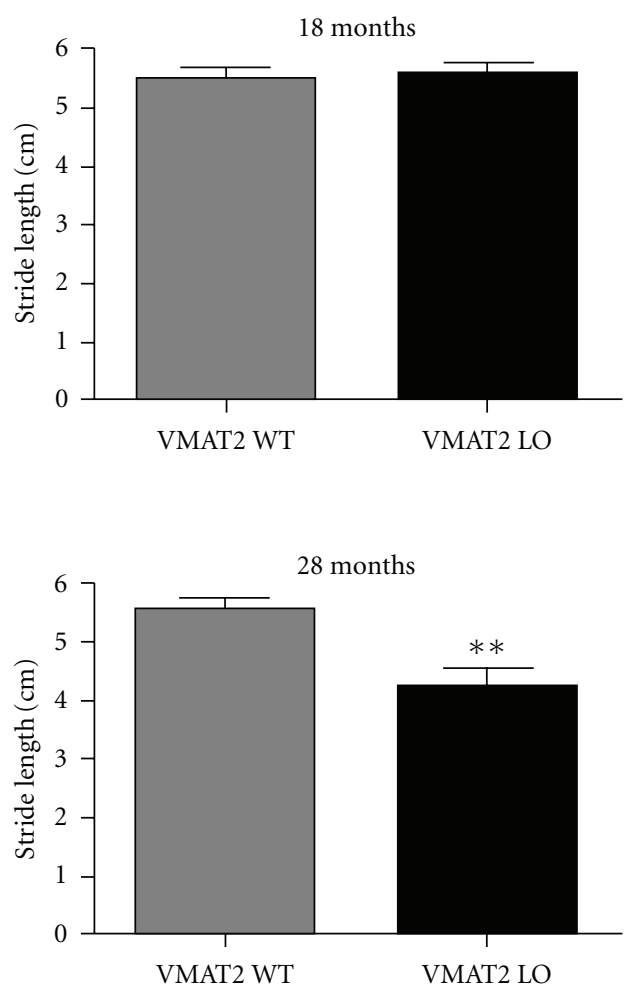

(a)
12 months

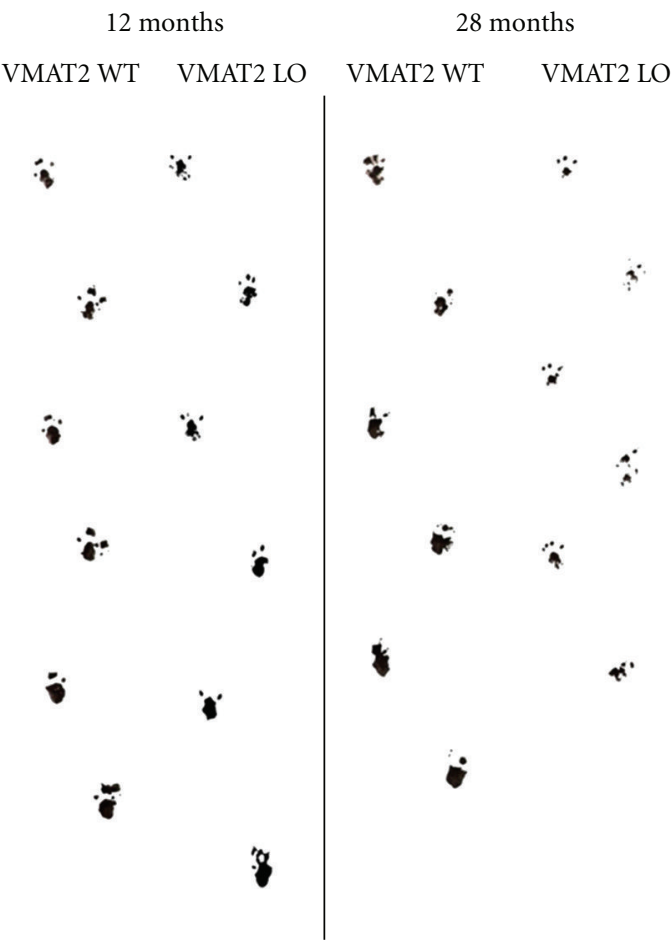

(b)

Figure 1: VMAT2-deficient animals display impaired stride length at older ages. (a) No deficits in forepaw stride length were apparent at 12 or 18 months of age in VMAT2-deficient mice. At 28 months of age, VMAT2-deficient mice display motor deficits as measured by inked paw stride length. Results represent average stride length $(\mathrm{cm}) \pm$ SEM for $4-6$ animals per genotype, $* * P<.01$. (b) Representative forepaw stride lengths for VMAT2 WT and deficient mice at 12 and 28 months of age.

$\alpha$-synuclein causes the downregulation of VMAT2 protein in vitro, triggering increases in cytosolic DA and ROS [33, 35]. Taken together with evidence from oxidative stress studies, these data demonstrate that the perturbation of VMAT2 can create an environment conducive to PD-related cell damage and pathology.

\section{Genetic Manipulation of VMAT2}

3.1. VMAT2 Knockout Mice. In order to investigate the exact role of VMAT2 in monoaminergic signaling several lines of transgenic VMAT2 mice have been generated. Unfortunately, complete deletion of the VMAT2 gene resulted in an animal, 
which moved little, fed poorly, and died within a few days after birth [36, 37], most likely a consequence of significantly reduced monoamine concentrations required for proper monoaminergic signaling. In light of this lethality, important information concerning the role of VMAT2 in monoamine neurotransmission was gleaned in vitro. For example, it was determined that VMAT2 is a key determinant of quantal release from monoaminergic cells, as levels were severely reduced or absent from transgenic cultures. Moreover, these data provided further evidence for the importance of presynaptic storage and release of monoamines for postsynaptic receptor responsiveness $[36,37]$.

Although the VMAT2 KO mice do not survive into adulthood, their creation also yielded mice that are heterozygous for VMAT2 (VMAT2 HT). Unlike the VMAT2 KO mice, the VMAT2 HTs are fully viable into adulthood, display a 50\% reduction in VMAT2 expression, and were physiologically similar to their wildtype littermates [38]. Although reports have varied, overall the VMAT2 HTs appear to have a significant reduction in monoamines, perceived to be a consequence of reduced vesicular storage capacity [3740]. Behaviorally, the VMAT2 HT mice perform normally in passive avoidance and locomotor activity tests, but display a depressive-like phenotype including anhedonia, locomotor retardation, and sensitivity to stress [38, 41]. This phenotype is ameliorated with the administration of antidepressants such as imipramine, fluoxetine, and bupropion, suggesting a combined involvement of all three monoamine neurotransmitters [41].

When challenged with various exogenous toxicants, the VMAT2 HTs begin to manifest deficits due to reduced vesicular storage capacity. Methamphetamine causes greater neurotoxicity in the VMAT2 HT mice compared to wild-type animals, with significant reductions in DA, DA metabolites, and DAT [42]. These findings were coupled with a less pronounced increase in extracellular DA, suggesting that cytosolic DA is the prevailing factor in the potentiation of methamphetamine toxicity observed in the mice [42]. Behaviorally, amphetamine produced enhanced locomotor activity but reduced reward as measured by conditioned place preference [38]. In addition to the amphetamines, VMAT2 HT mice were also found to be acutely more sensitive to the effects of the Parkinsonian drug MPTP. Presumably, due to their reduced capacity to sequester MPP+, VMAT2 HT mice undergo twice the dopaminergic cell loss observed in wild-type animals, accompanied by markers of striatal damage such as reductions in DA, DAT and increased glial fibrillary acidic protein (GFAP) mRNA $[38,39]$. Although the VMAT2 HT mice did not display any overt signs of Parkinsonism or PD-like neuropathology, they do exhibit an increased susceptibility to MPTP toxicity and thus, researchers postulated that the mice may be useful in teasing out the mechanisms of L-DOPA toxicity. It was found that primary DA neurons harvested from VMAT2 HTs were more vulnerable to L-DOPA than wildtype neurons; decreased VMAT2 activity might attenuate L-DOPA efficacy by augmenting endogenous dopaminergic toxicity [43]. However, these results were not observed in vivo [40]. Despite the absence of a clear link between vesicular storage and L-DOPA-induced dopaminergic dysfunction, manipulating VMAT2 still produces an increased sensitivity to parkinsonian toxins and signs of depression, one of the most prevalent nonmotor symptoms associated with PD.

3.2. The VMAT2 Hypomorph Mouse. As investigators continued to ponder the role of VMAT2 in the pathogenesis of $\mathrm{PD}$, further perturbation of the gene was necessary to produce a more profound disruption of monoamine storage than previously achieved with the VMAT2 HT mice. This perturbation was manifested in a line of mice that expressed only $5 \%$ of the VMAT2 protein. It is important to note that unlike the previous VMAT2 KO and HT mice, the KA1 line was created through gene targeting using a completely differently strain of mouse, which was found to be $\alpha$-synuclein null [44]. Both the hypomorphic VMAT2 allele and the $\alpha$-synuclein-null allele were both unintended consequences of an attempt to make VMAT2 knockout mice, but notably serendipitous to the PD field (see below). Unlike the VMAT2 KO mice, the KA1 mice are fully viable into adulthood with the absence of gross physical defects [45]. The survival of these KA1 mice allowed the examination of the effects of reduced vesicular storage over a lifetime, in addition to the study of the nuances of vesicular uptake mechanisms; whereas, both VMAT2 KO and chronically reserpinized animals are not amenable to studying the effects of aging on monoamine packaging defects.

Although no VMAT2 expression was detected in these mice through immunohistochemistry or in situ hybridization, residual VMAT2 was observed using western blotting approximating a $95 \%$ reduction $[10,45]$. Consequently, there were general reductions in tissue levels of the major monoamines, DA, NE, and 5-HT reduced by $92 \%, 87 \%$, and $82 \%$, respectively, which became progressively worse with age, accompanied by increased monoamine turnover and reduced DA availability in terminal and cell body regions of the SNpc and ventral tegmental area (VTA) $[45,46]$. In addition to the reduction of monoamines, the KAl mice were also found to have altered striatal neurotransmission and signaling. Although levels of DAT mRNA, protein, and activity and $D_{1} / D_{2}$ receptor expression remained unchanged, electrically stimulated DA release was dramatically reduced by approximately $70 \%$ compared to age-matched wild-type animals $[47,48]$. As demonstrated in the VMAT2 KO mice, a decrease in striatal DA release this dramatic is indicative of smaller vesicular DA stores due to a reduction in VMAT2 expression [47]. Considering that electrically stimulated DA release is absent in VMAT2 KO neurons, these data suggest considerable intraneuronal compensation for the $95 \%$ deficit in VMAT2 [37]. Moreover, due to the disproportionate decrease in DA release compared to the reduction in VMAT2 expression, it is possible to conceive that in wild-type neurons, not all VMAT2 protein is required to fill vesicles for exocytotic release; many transporters may, in fact, act as a reserve [47]. Additionally, even though no compensation was seen through changes in DA receptor expression, ablating VMAT2 by $95 \%$ did induce a supersensitization of the $D_{2} / D_{3}$ autoreceptors and downregulated phosphorylation of tyrosine hydroxylase (TH) at serine residues (Ser19, Ser31, and 
Ser40), which are critical for catechol feedback inhibition [46]. Finally, the KA1 mice were found to downregulate substance $\mathrm{P}$ while upregulating enkephalin, allowing for the possibility of abnormalities in organization of DA-mediated signaling via both the direct and indirect pathways $[45,48]$. Taken together, these data provide further evidence for the role of VMAT2 expression in regulating the size of both vesicular and cytosolic DA pools within the CNS, thus influencing extracellular neurotransmission $[46,47]$.

With the abundance of changes in striatal neurotransmission, the KA1 mice were tested for the presence of a behavioral phenotype that correlated with PD. As in reserpinized animals, reductions in VMAT2 in the KA1 mice cause a general decrease in locomotor activity [45]. At an early age, the KA1 mice demonstrated a significant impairment in motor coordination, independent of motivational factors, as measured by the challenging beam traversal and rotarod, which becomes progressively more severe with age $[45,46]$. However, they exhibit normal reactivity in novelty place preference task [45]. As expected, the KA1 are exquisitely sensitive to acute doses of MPTP and amphetamine. When exposed to amphetamine, the KA1 mice display an increase in stereotypic behaviors and abnormalities in DA release [45, 47]. Similarly, the KA1 have a lower threshold to MPTP toxicity, demonstrating dopaminergic damage and locomotor deficits [45]. Conversely, when L-DOPA is administered, the KA1 mice exhibit locomotor hyperactivity and amelioration of deficits in motor coordination and balance $[45,46]$. Interestingly, despite the presence of both striatal dopamine deficiency and a motor phenotype, when assessed for signs of Parkinsonian degeneration, no evidence of DA cell loss was found at any age [46]. However, as mentioned above, these mice contain a spontaneous chromosomal deletion spanning the $\alpha$-synuclein gene locus [44-47]. The lack of this noteworthy gene may account for the absence of degeneration as cytosolic dopamine and other monoamines have been proposed to inhibit $\alpha$-synuclein fibrillization by oxidatively ligating to $\alpha$-synuclein $[49,50]$, thus retaining $\alpha$-synuclein in its neurotoxic protofibril conformation. Assuming that protofibrils are the pathogenic species, a 95\% decrease of VMAT2 in neurons should have lethal implications, causing the cytosolic auto-oxidation of catecholamines to increase, amplifying protofibril concentration. To answer the question more fully, it was necessary to introduce the $\alpha$-synuclein gene into the mice with low VMAT2 expression.

3.3. Perfected Perturbation: VMAT2-Deficient Mice. Although the availability of the VMAT2 KA1 mice provided an extremely useful model with which to further examine the importance of DA handling, the complete ablation of such a ubiquitous protein such as $\alpha$-synuclein severely limited the utility of these mice from the perspective of dopamine handling and PD pathogenesis. Fortunately, the Emory colony of KA1 mice contained animals that were heterozygous for both the VMAT2 and $\alpha$-synuclein alleles. Through diligent breeding, all traces of the $\alpha$-synuclein mutation were eliminated from the KA1 line of mice yielding the VMAT2deficient mice. Consistent with previous reports of genetic and pharmacological reductions of VMAT2, striatal DA levels were reduced by $85 \%$ in VMAT2-deficient mice with a concomitant reduction in the metabolites, DOPAC and HVA; VMAT2-deficient mice also exhibited an age-dependent decline in DA [10]. Several intraneuronal compensatory mechanisms were also observed in the VMAT2-deficient mice including an increase in $\mathrm{TH}$ activity, increased DA turnover, and an age-dependent decline in DAT expression and activity [10]. Additionally, several markers of oxidative stress and damage were observed in the VMAT2-deficient mice. Although cysteinyl-DA was undetectable due to the reduced basal levels of DA and increased DA turnover, free cysteinyl-DOPA and DOPAC adducts were significantly increased at both 2 and 12 months of age; protein carbonyls and 3-nitrotyrosine did not manifest until 12 months of age, demonstrating that neuronal oxidative stress became progressively worse with age [10]. The chronic dysregulation of DA within VMAT2-deficient neurons began to contribute to neuronal degeneration in older animals, as evidence of cell death was seen through silver deposition and a progressive loss of TH-positive neurons within the SNpc [10].

Behaviorally, VMAT2-deficient mice exhibit many of the Parkinsonian motor phenotypes. Beginning at 2 months of age, VMAT2-deficient mice have general deficits in noveltyinduced locomotor activity, which is L-DOPA responsive (Table 1) [10]. Interestingly, in the VMAT2-deficient mice it has been observed that the major motor deficits do not appear until 28 months of age, coinciding with the most severe nigral cell loss (Taylor and Miller, unpublished observations). Compared to age-matched wild-type littermates, VMAT2-deficient mice do not demonstrate a deficit in forepaw stride length until 28 months of age; this behavior is thought to mimic the shuffling gait observed in PD patients [51] (Figure 1). This behavior is also L-DOPA responsive, establishing that the motor phenotype is due to dopamine insufficiency. Combined with the dopaminergic characterization of these mice, these data reveal that reduced vesicular storage of DA is enough to induce Parkinsonian neurodegeneration.

Mounting evidence for degeneration of the locus coeruleus (LC) in human PD highlights the importance of expanding the focus of research from the nigrostriatal system in order to expose the deficits in other neurotransmitter systems [3, 7, 52-55]. Beginning at 18 months of age, the VMAT2-deficient mice displayed a mild reduction in $\mathrm{TH}$ staining in the SNpc and striatum, which increased moderately with age $[10,56]$. More dramatic reductions in $\mathrm{TH}$ staining were observed in the locus coeruleus (LC) at 18, 24, and 30 months of age [56]. This pattern of neuronal loss was verified using unbiased stereological counts, demonstrating that neuronal loss in the LC precedes nigral loss in the VMAT2-deficient mice [56]. The LC of VMAT2-deficient mice undergoes a much more rapid decline from 12 to 18 months of age, with an overall $72 \%$ neuronal loss from 630 months of age [56]. The SNpc of the VMAT2-deficient mice does not start to degenerate until 18 months of age, with an overall 59\% cell loss, similar to the loss observed in humans [56]. Taken together, these data suggest that, unlike other chemical and genetic models of PD, the LC undergoes 
a much more severe degeneration than the SNpc in the VMAT2-deficient mice.

In classical PD, motor disturbances do not present clinically until approximately $70-80 \%$ of striatal dopamine and $40-50 \%$ of nigral cell bodies have already been lost; however, other nonmotor symptoms are evident before the onset of motor disturbances. These include, but are not limited to, hyposmia/anosmia, gastrointestinal disturbances, sleep abnormalities, autonomic dysfunction, anxiety, and depression [52, 57]. It is probable that other neurotransmitters such as NE and 5-HT significantly contribute to these symptoms, as both the $\mathrm{LC}$ and raphe nucleus have also been shown to degenerate in $\mathrm{PD}$, in addition to the SNpc $[3,54,55]$. With the pathology observed in the major monoaminergic systems of the VMAT2-deficient mice, the presence of nonmotor phenotypes would not be unlikely.

Olfactory disturbances are one of the first nonmotor symptoms observed in PD; patients have demonstrated impairments in odor detection, differentiation, and identification [58-60]. Moreover, this nonmotor symptom is not responsive to traditional dopaminergic therapies [61]. When subjected to a battery of olfactory discrimination tests at various ages, VMAT2-deficient animals were unable to discriminate between two blocks (one scented with bedding from their home cage and one scented from the cage of a foreign animal of the same sex), and consequently displayed no preferential exploration of either block [62]. VMAT2 wild-type animals displayed preferential exploration of the foreign-scented block at all ages tested [62]. When challenged in a similar test of olfactory acuity using scents commonly used on the University of Pennsylvania Smell Identification Test (UPSIT), VMAT2-deficient mice again showed no preferential exploration of the novel scent as compared with water, whereas VMAT2 wild-type animals spent more time investigating the novel scent [62]. The olfactory deficit is not corrected by L-DOPA treatment in human PD patients, nor is it effective in our mice (Table 1). To ensure there was not a problem in general sensory perception, mice were tested for nonolfactory sensory deficits. VMAT2-deficient animals showed no deficits in response to tactile stimulation, quinine taste aversion, trigeminal nerve function, muscle strength, or visual acuity [62].

In order to investigate behavioral sleep disturbances in the VMAT2-deficient mice, sleep latency tests were conducted in VMAT2 wild-type and deficient mice during their circadian nadir. Beginning at 2 months of age, VMAT2deficient mice show a shorter latency to behavioral signs of sleep compared to age-matched wild-type controls, which is responsive to an acute dose of L-DOPA (Table 1) [62]. The circadian activity of VMAT2-deficient animals is also significantly lower than that of age-matched wild-type controls at younger ages, but follows normal patterns compared to wild-type animals [62]. VMAT2-deficient animals were next behaviorally examined for gastric emptying at 2, 6, 12, and 18 months of age, as gastrointestinal dysfunction in PD occurs in over $70 \%$ of PD patients $[9,57]$. Solid gastric emptying was significantly delayed overall in VMAT2-deficient mice, with an increased stool frequency, indicating a fair amount of gastrointestinal dysfunction in the VMAT2-deficient animals
TABLE 1: Summary of L-DOPA responsive Parkinsonian symptoms.

\begin{tabular}{lcc}
\hline Behavior & $\begin{array}{c}\text { L-DOPA } \\
\text { responsive in } \\
\text { VMAT2-Deficient? }\end{array}$ & $\begin{array}{c}\text { L-DOPA } \\
\text { responsive in } \\
\text { humans? }\end{array}$ \\
\hline $\begin{array}{l}\text { Olfactory Discrimination } \\
\text { Sleep Latency }\end{array}$ & No & No \\
Anxiety & Yes & No \\
$\begin{array}{l}\text { Depression } \\
\text { Gastrointestinal }\end{array}$ & Yes & Variable \\
$\begin{array}{l}\text { Dysfunction } \\
\text { Locomotor Activity }\end{array}$ & No & Variable \\
Forepaw Stride Length & Yes & No \\
\hline
\end{tabular}

*Falling, freezing of gait, and postural instability are all L-DOPA unresponsive.

[62]. As in humans, an acute dose of L-DOPA did not ameliorate the gastrointestinal dysfunction observed in these animals (Table 1).

Disruptions in DA, NE, and 5-HT neurotransmission, including degeneration of the LC and DR, have been found in PD patients with anxiety and/or depression; similar pathology has been observed in the VMAT2-deficient mice indicating the possibility for both anxiety-like and depressivelike phenotypes $[9,53,62]$. Moreover, the VMAT2 HT mice have been previously found to display a depressive-like phenotype [41]. Severe reduction of VMAT2 expression in the VMAT2-deficient mice was found to trigger both anxiety and progressive depressive behavior. VMAT2-deficient mice showed a significant increase in percentage of open arm time in the elevated plus maze at 4-6 months of age, while the increased immobility time in the forced swim and tail suspension tests did not occur until 12 months of age; suggesting that anxiety precedes depressive symptoms in VMAT2deficient animals and that the depressive-like phenotype is progressive [62]. Additionally, a low dose of desipramine that had no effect in wild-type animals was able to normalize immobility times in VMAT2-deficient mice; similarly, an acute dose of L-DOPA was also able to ameliorate depressivelike symptoms in the VMAT2-deficient mice (Table 1) [62]. Despite the presence of many of the nonmotor symptoms associated with PD in the VMAT2-deficient animals, the animals have not yet been tested for cognitive deficits or presence of autonomic dysfunction. The involvement of multiple neurotransmitter systems and evidence from other mouse models with noradrenergic degeneration suggests that cognitive and cardiovascular deficit may also be present in the VMAT2-deficient animals $[63,64]$.

\section{Conclusions}

As the VMAT2-deficient mice have reduced levels of DA, NE, and 5-HT, L-DOPA responsive motor deficits, and almost the full constellation of nonmotor symptoms, mice with altered VMAT2 expression may represent a new model of PD that encompasses many of the motor and nonmotor symptoms, as well as the neurochemical pathophysiology (Figure 2) [10, 41, 45, 62]. Moreover, most current models 


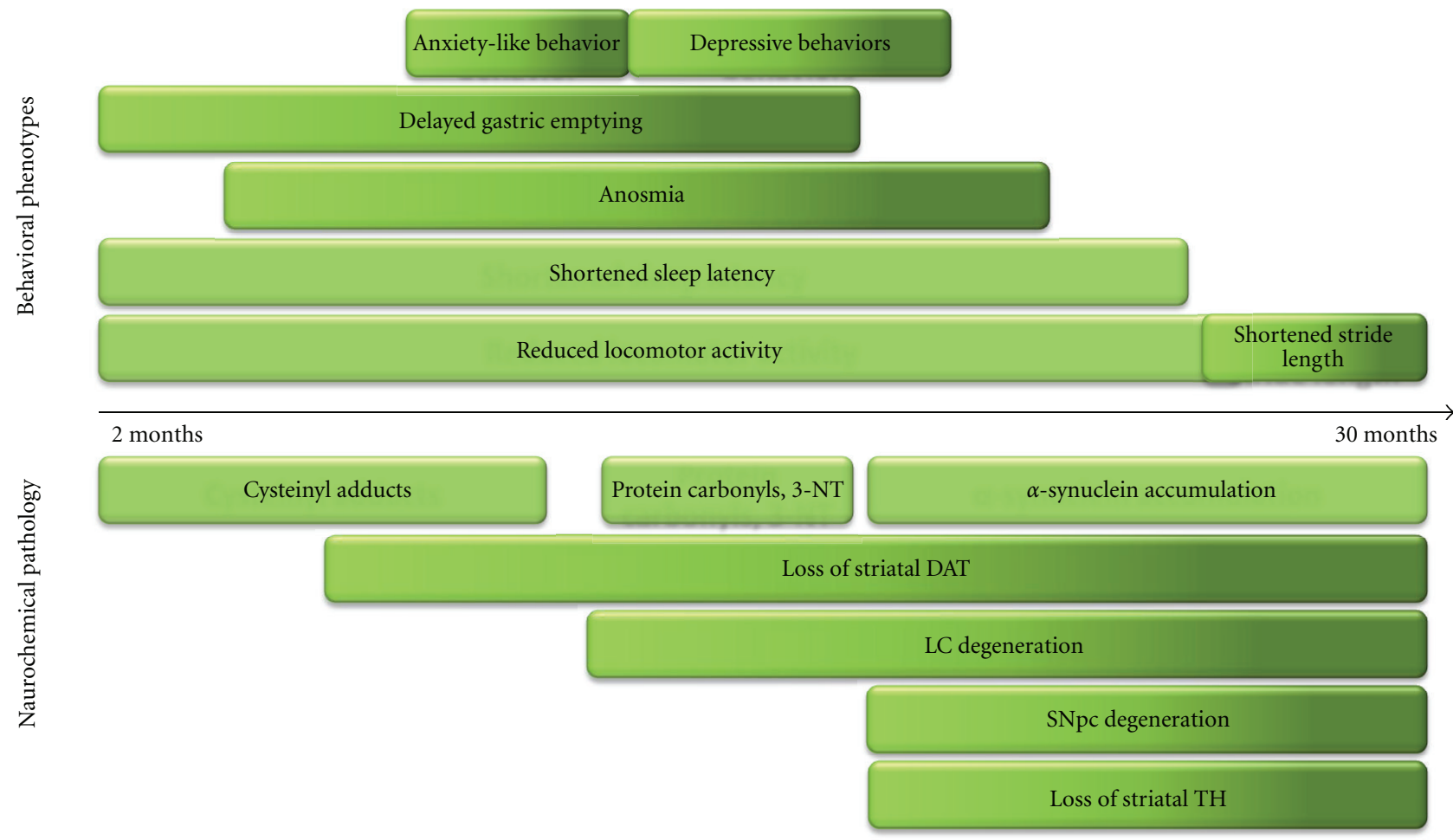

FIgure 2: Timeline of Parkinsonian features observed in the VMAT2-deficient mice from 2-30 months of age. Symptoms or pathology indicated by a solid colored box did not increase in severity as the mice aged. All boxes end at the last age the symptom or pathology was measured. Behavioral phenotypes: reductions in locomotor activity and latency to behavioral signs of sleep were first observed at 2 months of age and were found to persist until their last measurements at 6 months of age and 18 months of age, respectively. Gastric emptying was first measured at 2 months of age, and increased in severity until the last time point at 18 months of age. Hyposmia began at 4 months, with full anosmia at 6 months of age and persisting until the last evaluation at 18 months. Anxiety-like behavior was first assessed at 4 months of age, persisting until 6 months of age. Even though depressive-like behaviors were measured at 4-6 months of age, presence of a depressive-like phenotype was not detected until 12 months of age, lasting until 15 months of age. Finally, reductions in forepaw stride length were not seen until 27 months, reaching full severity at 30 months of age. Neurochemical pathology: evidence of oxidative damage was first observed through the formation of cysteinyl adducts at 2 months, which were still present at 12 months. Protein carbonyls and 3-nitrotyrosine formation did not occur until 12 months of age. Accumulation of $\alpha$-synuclein began at 18 months with evidence remaining until 24 months of age. Loss of striatal DAT expression measured immunohistochemically began at 6 months of age progressing in severity until 22 months of age. Reductions in striatal TH expression begin at 18 months of age, reaching maximal severity at 30 months. Degeneration of the LC starts at 12 months of age in the VMAT2-deficient animals, preceding nigral loss, which does not begin until 18 months of age.

of $\mathrm{PD}$, genetic and chemical, represent a relatively short disease progression. The average lifespan of a mouse is two years; disease progression must reflect this because sporadic PD, like Alzheimer's disease, is a disease of aging. The VMAT2-deficient mice exhibit a high age dependency coupled with a progressive behavioral decline (Figure 2). The nigral and extranigral pathology combined with the motor and nonmotor symptoms in the VMAT2-deficient mice strongly argue that the underlying pathogenesis of human PD likely has some common features. For example, many of the PARK genes have been shown to disrupt proper recycling, trafficking, and release of vesicles. While the mode of vesicular disturbance may differ in individual PD cases, disrupted vesicular function, whether it is via storage or trafficking, of monoamines may represent a common pathogenic mechanism. These mice demonstrate that it is possible that PD pathogenesis represents more than altered DA homeostasis; a global disruption of monoamine storage and handling may be necessary to fully invoke the pathology associated with the disease. Utilizing the VMAT2-deficient mice as a new model of PD, could potentially lead to new adjunct therapeutic strategies, which complements current dopamine replacement therapy, improving the quality of life for many patients.

\section{References}

[1] J. Parkinson, An Essay on the Shaking Palsy, Sherwood, Neely, and Jones, London, UK, 1817.

[2] C. W. Olanow and W. G. Tatton, "Etiology and pathogenesis of Parkinson's disease," Annual Review of Neuroscience, vol. 22, pp. 123-144, 1999.

[3] S. Fahn and D. Sulzer, "Neurodegeneration and neuroprotection in Parkinson disease," NeuroRx, vol. 1, no. 1, pp. 139-154, 2004. 
[4] M. Saito, M. Maruyama, K. Ikeuchi et al., "Autosomal recessive juvenile parkinsonism," Brain and Development, vol. 22, supplement 1, pp. S115-S117, 2000.

[5] K. Jellinger, "New developments in the pathology of Parkinson's disease," Advances in Neurology, vol. 53, pp. 1-16, 1990.

[6] P. Jenner and C. W. Olanow, "The pathogenesis of cell death in Parkinson's disease," Neurology, vol. 66, no. 10, supplement 4, pp. S24-S30, 2006.

[7] H. Braak, K. Del Tredici, H. Bratzke, J. Hamm-Clement, D. Sandmann-Keil, and U. Rüb, "Staging of the intracerebral inclusion body pathology associated with idiopathic Parkinson's disease (preclinical and clinical stages)," Journal of Neurology, Supplement, vol. 249, supplement 3, pp. III1-III5, 2002.

[8] ED. G. Gonera, M. Van't Hof, H. J. C. Berger, C. Van Weel, and M. W. I. M. Horstink, "Symptoms and duration of the prodromal phase in Parkinson's disease," Movement Disorders, vol. 12, no. 6, pp. 871-876, 1997.

[9] T. Ziemssen and H. Reichmann, "Non-motor dysfunction in Parkinson's disease," Parkinsonism and Related Disorders, vol. 13, no. 6, pp. 323-332, 2007.

[10] W. M. Caudle, J. R. Richardson, M. Z. Wang et al., "Reduced vesicular storage of dopamine causes progressive nigrostriatal neurodegeneration," Journal of Neuroscience, vol. 27, no. 30, pp. 8138-8148, 2007.

[11] P. Jenner, . Hunot, . Olanow et al., "Oxidative stress in Parkinson's disease," Annals of Neurology, vol. 53, no. 3, pp. S26-S38, 2003.

[12] C. K. Surratt, A. M. Persico, D. X. Yang et al., "A human synaptic vesicle monoamine transporter $\mathrm{CDNA}$ predicts posttranslational modifications, reveals chromosome 10 gene localization and identifies TaqI RFLPs," FEBS Letters, vol. 318, no. 3, pp. 325-330, 1993.

[13] J. D. Erickson, L. E. Eiden, and B. J. Hoffman, "Expression cloning of a reserpine-sensitive vesicular monoamine transporter," Proceedings of the National Academy of Sciences of the United States of America, vol. 89, no. 22, pp. 10993-10997, 1992.

[14] Y. Liu, D. Peter, A. Roghani et al., "A cDNA that suppresses MPP toxicity encodes a vesicular amine transporter," Cell, vol. 70, no. 4, pp. 539-551, 1992.

[15] M. Forgac, "Structure and function of vacuolar class of ATPdriven proton pumps," Physiological Reviews, vol. 69, no. 3, pp. 765-796, 1989.

[16] G. Rudnick, "ATP-driven $\mathrm{H}$ pumping into intracellular organelles," Annual Review of Physiology, vol. 48, pp. 403-413, 1986.

[17] L. E. Eiden, M. K. H. Schäfer, E. Weihe, and B. Schütz, "The vesicular amine transporter family (SLC18): amine/proton antiporters required for vesicular accumulation and regulated exocytotic secretion of monoamines and acetylcholine," Pflugers Archiv, vol. 447, no. 5, pp. 636-640, 2004.

[18] S. Schuldiner, A. Shirvan, and M. Linial, "Vesicular neurotransmitter transporters: from bacteria to humans," Physiological Reviews, vol. 75, no. 2, pp. 369-392, 1995.

[19] E. Vardy, I. T. Arkin, K. E. Gottschalk, H. R. Kaback, and S. Schuldiner, "Structural conservation in the major facilitator superfamily as revealed by comparative modeling," Protein Science, vol. 13, no. 7, pp. 1832-1840, 2004.

[20] G. W. Miller, R. R. Gainetdinov, A. I. Levey, and M. G. Caron, "Dopamine transporters and neuronal injury," Trends in Pharmacological Sciences, vol. 20, no. 10, pp. 424-429, 1999.
[21] D. G. Graham, "Oxidative pathways for catecholamines in the genesis of neuromelanin and cytotoxic quinones," Molecular Pharmacology, vol. 14, no. 4, pp. 633-643, 1978.

[22] E. D. Freis, "Mental depression in hypertensive patients treated for long periods with large doses of reserpine," The New England Journal of Medicine, vol. 251, no. 25, pp. 1006-1008, 1954.

[23] D. Peter, J. Jimenez, Y. Liu, J. Kim, and R. H. Edwards, "The chromaffin granule and synaptic vesicle amine transporters differ in substrate recognition and sensitivity to inhibitors," Journal of Biological Chemistry, vol. 269, no. 10, pp. 72317237, 1994.

[24] J. A. Schneider, "Reserpine antagonism of morphine analgesia in mice," Proceedings of the Society for Experimental Biology and Medicine, vol. 87, no. 3, pp. 614-615, 1954.

[25] P. O. Fernagut, E. Diguet, B. Labattu, and F. Tison, "A simple method to measure stride length as an index of nigrostriatal dysfunction in mice," Journal of Neuroscience Methods, vol. 113, no. 2, pp. 123-130, 2002.

[26] L. L. Skalisz, V. Beijamini, S. L. Joca, M. A. B. F. Vital, C. Da Cunha, and R. Andreatini, "Evaluation of the face validity of reserpine administration as an animal model of depression-Parkinson's disease association," Progress in NeuroPsychopharmacology and Biological Psychiatry, vol. 26, no. 5, pp. 879-883, 2002.

[27] R. H. Silva, V. C. Abílio, D. Torres-Leite et al., "Concomitant development of oral dyskinesia and memory deficits in reserpine-treated male and female mice," Behavioural Brain Research, vol. 132, no. 2, pp. 171-177, 2002.

[28] G. R. Uhl, "Hypothesis: the role of dopaminergic transporters in selective vulnerability of cells in Parkinson's disease," Annals of Neurology, vol. 43, no. 5, pp. 555-560, 1998.

[29] Y. Liu and R. H. Edwards, "The role of vesicular transport proteins in synaptic transmission and neural degeneration," Annual Review of Neuroscience, vol. 20, pp. 125-156, 1997.

[30] M. R. Kilbourn, J. N. DaSilva, K. A. Frey, R. A. Koeppe, and D. E. Kuhl, "In vivo imaging of vesicular monoamine transporters in human brain using [C] tetrabenazine and positron emission tomography," Journal of Neurochemistry, vol. 60, no. 6, pp. 2315-2318, 1993.

[31] G. W. Miller, J. D. Erickson, J. T. Perez et al., "Immunochemical analysis of vesicular monoamine transporter (VMAT2) protein in Parkinson's disease," Experimental Neurology, vol. 156, no. 1, pp. 138-148, 1999.

[32] C. E. Glatt, A. D. Wahner, D. J. White, A. Ruiz-Linares, and B. Ritz, "Gain-of-function haplotypes in the vesicular monoamine transporter promoter are protective for Parkinson disease in women," Human Molecular Genetics, vol. 15, no. 2, pp. 299-305, 2006.

[33] J. Lotharius and P. Brundin, "Pathogenesis of Parkinson's disease: dopamine, vesicles and alpha-synuclein," Nature reviews. Neuroscience, vol. 3, no. 12, pp. 932-942, 2002.

[34] J. T. Guo, AN. Q. Chen, QI. Kong, H. Zhu, C. M. Ma, and C. Qin, "Inhibition of vesicular monoamine transporter2 activity in $\alpha$-synuclein stably transfected SH-SY5Y cells," Cellular and Molecular Neurobiology, vol. 28, no. 1, pp. 35-47, 2008.

[35] E. V. Mosharov, R. G. W. Staal, J. Bové et al., " $\alpha$-Synuclein overexpression increases cytosolic catecholamine concentration," Journal of Neuroscience, vol. 26, no. 36, pp. 9304-9311, 2006.

[36] E. A. Fon, E. N. Pothos, B. C. Sun, N. Killeen, D. Sulzer, and R. H. Edwards, "Vesicular transport regulates monoamine storage and release but is not essential for amphetamine action," Neuron, vol. 19, no. 6, pp. 1271-1283, 1997. 
[37] Y. M. Wang, R. R. Gainetdinov, F. Fumagalli et al., "Knockout of the vesicular monoamine transporter 2 gene results in neonatal death and supersensitivity to cocaine and amphetamine," Neuron, vol. 19, no. 6, pp. 1285-1296, 1997.

[38] N. Takahashi, L. L. Miner, I. Sora et al., "VMAT2 knockout mice: heterozygotes display reduced amphetamineconditioned reward, enhanced amphetamine locomotion, and enhanced MPTP toxicity," Proceedings of the National Academy of Sciences of the United States of America, vol. 94, no. 18, pp. 9938-9943, 1997.

[39] R. R. Gainetdinov, F. Fumagalli, Y. M. Wang et al., "Increased MPTP neurotoxicity in vesicular monoamine transporter 2 heterozygote knockout mice," Journal of Neurochemistry, vol. 70, no. 5, pp. 1973-1978, 1998.

[40] M. E. Reveron, K. V. Savelieval, J. L. Tillerson, A. L. McCormack, D. A. Di Monte, and G. W. Miller, "L-DOPA does not cause neurotoxicity in VMAT2 heterozygote knockout mice," NeuroToxicology, vol. 23, no. 4-5, pp. 611-619, 2002.

[41] M. Fukui, R. M. Rodriguiz, J. Zhou et al., "Vmat2 heterozygous mutant mice display a depressive-like phenotype," Journal of Neuroscience, vol. 27, no. 39, pp. 10520-10529, 2007.

[42] F. Fumagalli, R. R. Gainetdinov, Y. M. Wang, K. J. Valenzano, G. W. Miller, and M. G. Caron, "Increased methamphetamine neurotoxicity in heterozygous vesicular monoamine transporter 2 knock-out mice," Journal of Neuroscience, vol. 19, no. 7, pp. 2424-2431, 1999.

[43] S. Kariya, N. Takahashi, M. Hirano, and S. Ueno, "Increased vulnerability to L-DOPA toxicity in dopaminergic neurons from VMAT2 heterozygote knockout mice," Journal of Molecular Neuroscience, vol. 27, no. 3, pp. 277-279, 2005.

[44] C. G. Specht and R. Schoepfer, "Deletion of the alphasynuclein locus in a subpopulation of C57BL/6 $\mathrm{J}$ inbred mice," BMC Neuroscience, vol. 2, article 11, 2001.

[45] K. A. Mooslehner, . Pok Man Chan, W. Xu et al., "Mice with very low expression of the vesicular monoamine transporter 2 gene survive into adulthood: potential mouse model for parkinsonism," Molecular and Cellular Biology, vol. 21, no. 16, pp. 5321-5331, 2001.

[46] R. E. Colebrooke, T. Humby, P. J. Lynch, D. P. McGowan, J. Xia, and P. C. Emson, "Age-related decline in striatal dopamine content and motor performance occurs in the absence of nigral cell loss in a genetic mouse model of Parkinson's disease," European Journal of Neuroscience, vol. 24, no. 9, pp. 2622-2630, 2006.

[47] J. Patel, K. A. Mooslehner, P. M. Chan, P. C. Emson, and J. A. Stamford, "Presynaptic control of striatal dopamine neurotransmission in adult vesicular monoamine transporter 2 (VMAT2) mutant mice," Journal of Neurochemistry, vol. 85, no. 4, pp. 898-910, 2003.

[48] R. E. Colebrooke, P. M. Chan, P. J. Lynch, K. Mooslehner, and P. C. Emson, "Differential gene expression in the striatum of mice with very low expression of the vesicular monoamine transporter type 2 gene," Brain Research, vol. 1152, no. 1, pp. 10-16, 2007.

[49] E. H. Norris, B. I. Giasson, R. Hodara et al., "Reversible inhibition of $\alpha$-synuclein fibrillization by dopaminochromemediated conformational alterations," Journal of Biological Chemistry, vol. 280, no. 22, pp. 21212-21219, 2005.

[50] K. A. Conway, J. C. Rochet, R. M. Bieganski, and P. T. Lansbury, "Kinetic stabilization of the $\alpha$-synuclein protofibril by a dopamine- $\alpha$-synuclein adduct," Science, vol. 294 , no. 5545, pp. 1346-1349, 2001.

[51] J. L. Tillerson, W. M. Caudle, M. E. Reverón, and G. W. Miller, "Detection of behavioral impairments correlated to neurochemical deficits in mice treated with moderate doses of 1-methyl-4-phenyl-1,2,3,6-tetrahydropyridine," Experimental Neurology, vol. 178, no. 1, pp. 80-90, 2002.

[52] H. Braak, K. Del Tredici, U. Rüb, R. A. I. De Vos, E. N. H. Jansen Steur, and E. Braak, "Staging of brain pathology related to sporadic Parkinson's disease," Neurobiology of Aging, vol. 24, no. 2, pp. 197-211, 2003.

[53] M. R. Lemke, G. Fuchs, I. Gemende et al., "Depression and Parkinson's disease," Journal of Neurology, Supplement, vol. 251, supplement 6, pp. VI/24-VI/27, 2004.

[54] W. Dauer and S. Przedborski, "Parkinson's disease: mechanisms and models," Neuron, vol. 39, no. 6, pp. 889-909, 2003.

[55] K. S. Rommelfanger and D. Weinshenker, "Norepinephrine: the redheaded stepchild of Parkinson's disease," Biochemical Pharmacology, vol. 74, no. 2, pp. 177-190, 2007.

[56] T. N. Taylor et al., "Progressive noradrenergic degeneration precedes nigral cell loss in a mouse model of Parkinson's disease," in Proceedings of the Annual Meeting of the Society for Neuroscience, San Diego, Calif, USA, 2010.

[57] J. W. Langston, “The Parkinson's complex: parkinsonism is just the tip of the Iceberg," Annals of Neurology, vol. 59, no. 4, pp. 591-596, 2006.

[58] R. L. Doty, M. B. Stern, C. Pfeiffer, S. M. Gollomp, and H. I. Hurtig, "Bilateral olfactory dysfunction in early stage treated and untreated idiopathic Parkinson's disease," Journal of Neurology Neurosurgery and Psychiatry, vol. 55, no. 2, pp. 138-142, 1992.

[59] G. Tissingh, J. Booij, P. Bergmans et al., "Iodine-123-N- $\omega$ fluoropropyl-2/ $\beta$-carbomethoxy $3 \beta$-(4-iodophenyl)tropane SPECT in healthy controls and early-stage, drug-naive Parkinson's disease," Journal of Nuclear Medicine, vol. 39, no. 7, pp. 1143-1148, 1998.

[60] C. D. Ward, W. A. Hess, and D. B. Calne, "Olfactory impairment in Parkinson's disease," Neurology, vol. 33, no. 7, pp. 943-946, 1983.

[61] S. M. Kranick and J. E. Duda, "Olfactory dysfunction in Parkinson's disease,” NeuroSignals, vol. 16, no. 1, pp. 35-40, 2007.

[62] T. N. Taylor, W. M. Caudle, K. R. Shepherd et al., "Nonmotor symptoms of Parkinson's disease revealed in an animal model with reduced monoamine storage capacity," Journal of Neuroscience, vol. 29, no. 25, pp. 8103-8113, 2009.

[63] S. J. Swoap, D. Weinshenker, R. D. Palmiter, and G. Garber, "Dbh(-/-) mice are hypotensive, have altered circadian rhythms, and have abnormal responses to dieting and stress," American Journal of Physiology, vol. 286, no. 1, pp. R108-R113, 2004.

[64] M. T. Heneka, M. Ramanathan, A. H. Jacobs et al., "Locus ceruleus degeneration promotes Alzheimer pathogenesis in amyloid precursor protein 23 transgenic mice," Journal of Neuroscience, vol. 26, no. 5, pp. 1343-1354, 2006. 


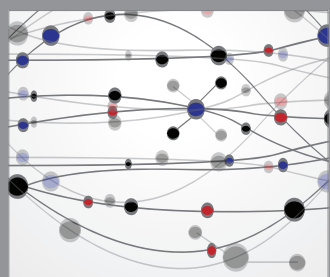

The Scientific World Journal
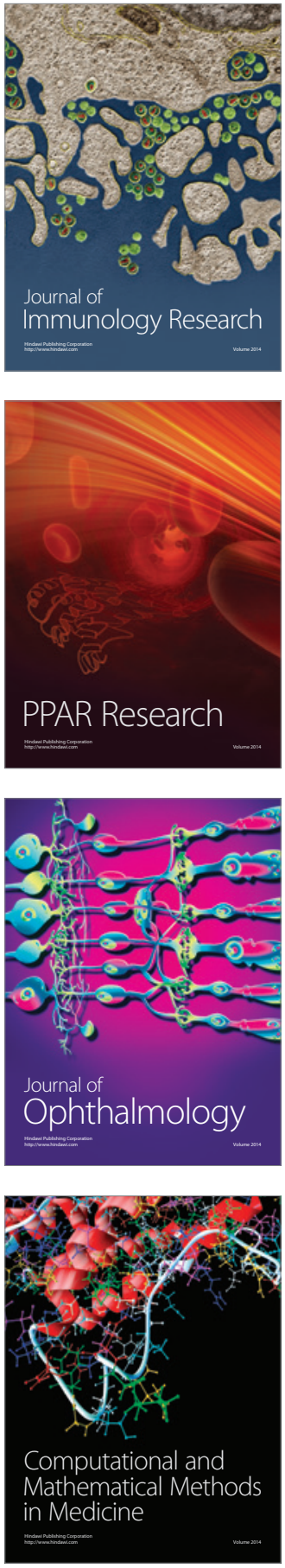

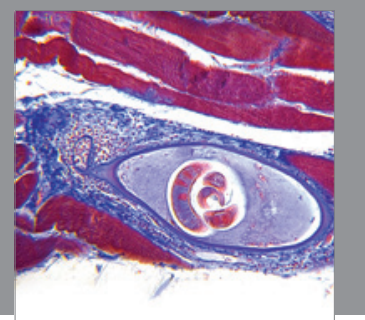

Gastroenterology

Research and Practice
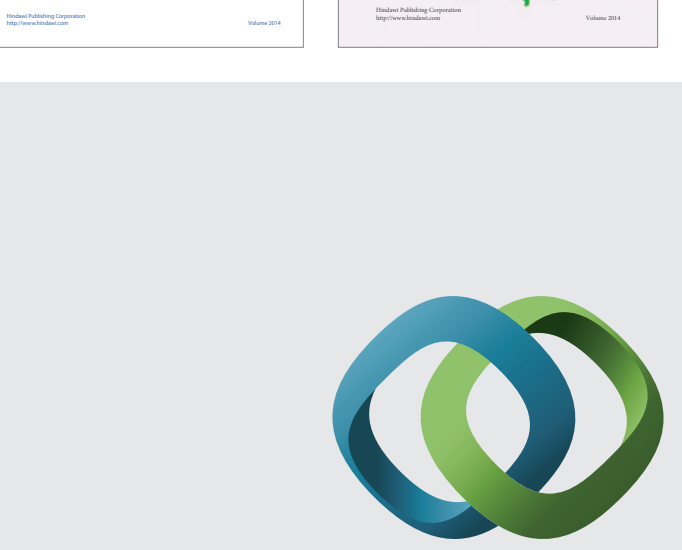

\section{Hindawi}

Submit your manuscripts at

http://www.hindawi.com
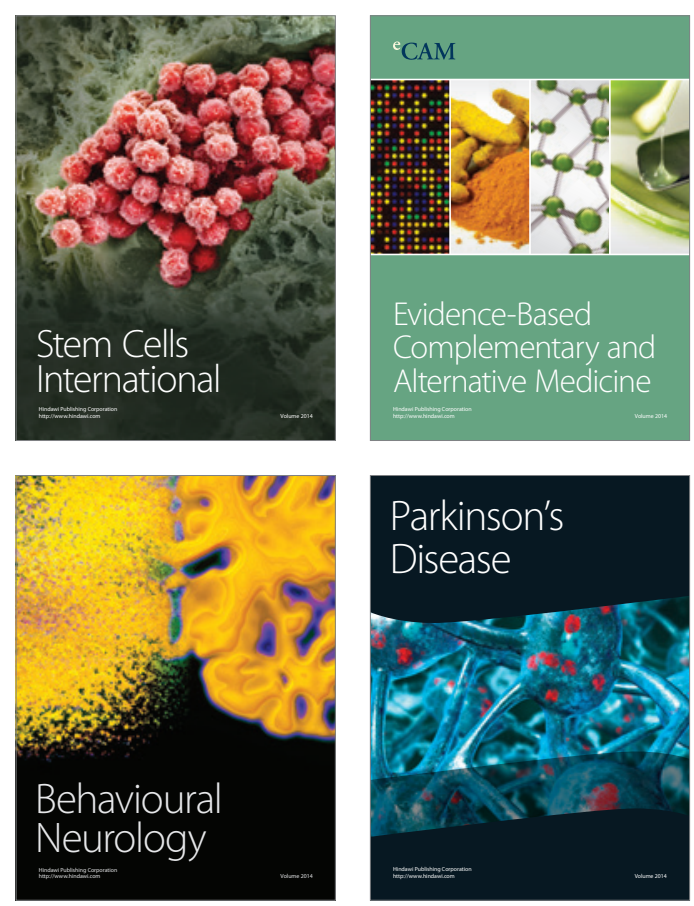

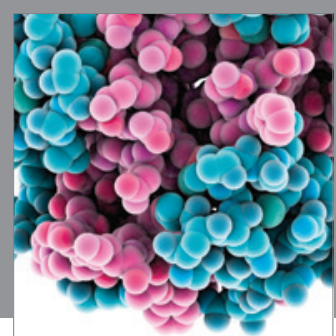

Journal of
Diabetes Research

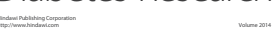

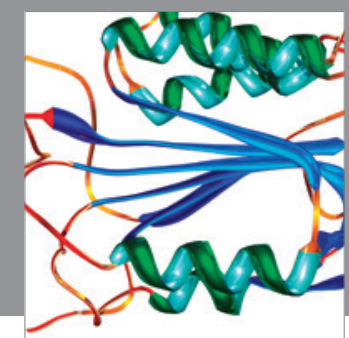

Disease Markers
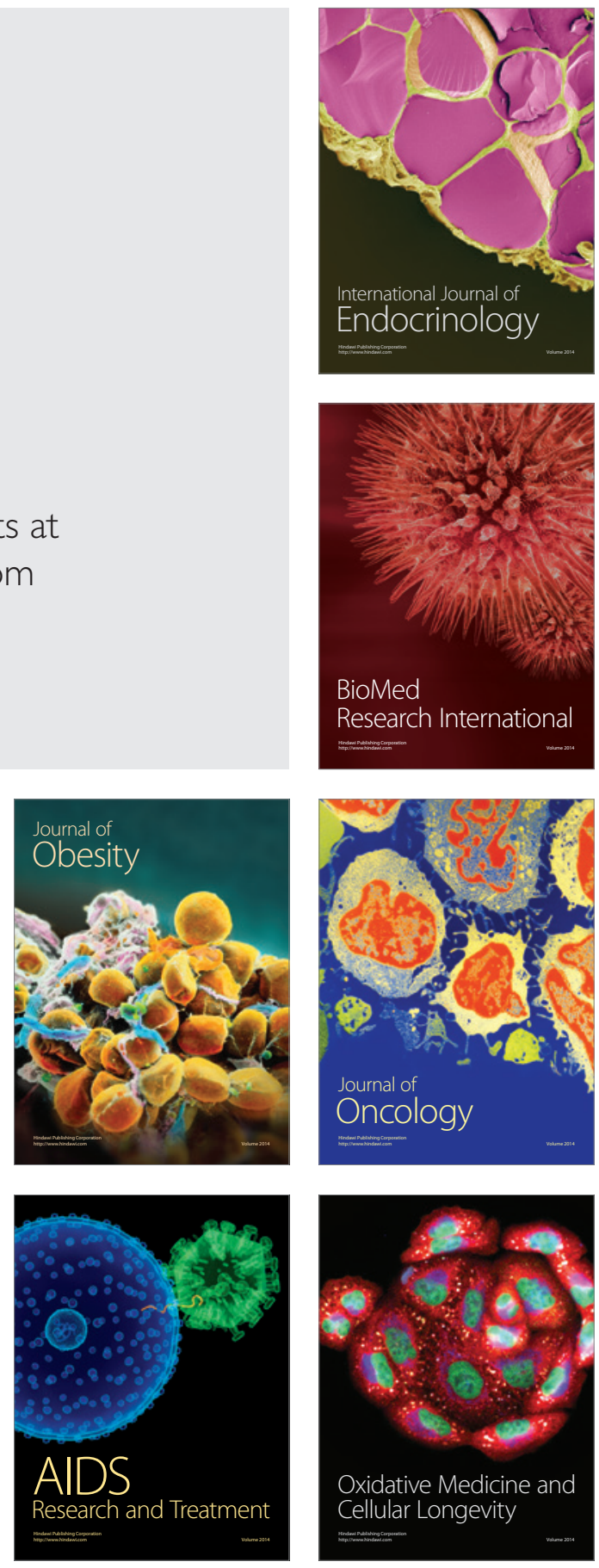